\title{
How to consider the Interfacial Transition Zones in the finite element modelling of concrete?
}

\author{
F. Grondin ${ }^{\mathrm{a}, *}$, M. Matallah ${ }^{\mathrm{a}, \mathrm{b}}$ \\ a Institut de Recherche en Génie Civil et Mécanique (GeM), UMR-CNRS 6183, Ecole Centrale de Nantes, 1 rue de la Noe, F-44321 Nantes, France \\ b RiSAM, University of Tlemcen, BP 230 Tlemcen, Algeria
}

Because the ITZs' thickness in concrete surrounding aggregates is tiny, it is difficult to take them into account explicitly in the modelling of concrete. A methodology is suggested to consider the effect of the ITZs on the mechanical behaviour of concrete at the mesoscopic scale. For that, the mechanical ITZ properties have been calculated according to the hydration of cement at various distances of the aggregate boundary. Then, we have defined an effective mixed interphase (EMI) around each aggregate which is formed by the ITZ and a volume fraction of the bulk cement paste. Simulations have been performed to show the influence of this EMI on the tensile failure of concrete. Results have shown that the maximum strength of the specimens depends on the local tensile strength of the ITZs. Stresses were more diffused for the cases without the ITZs and with the ITZs having a lower tensile strength.

\section{Introduction}

This study aims to suggest a method to consider the presence of the Interfacial Transition Zone (ITZ) around aggregates in concrete. The ITZs are the thin thicknesses of cement paste around aggregates with different properties than the cement paste which form the matrix of concrete (called the bulk cement paste). The main theory suggests that it is due to the cement grains in contact with the aggregate boundary at the moment of mixing (called the wall effect) which limit the water motion in this area and this water, after hydration of the cement clinkers, results in the formation of the porosity $[1,2]$. As a high quantity of water is maintained in this area, a high volume of porosity is created. The ITZ thickness $\left(\delta_{I T Z}\right)$ is between $20 \mu \mathrm{m}$ and $50 \mu \mathrm{m}$ and some experimental studies have shown that it corresponds to the mean radius of cement grains [1]. The value of the thickness and the ITZ properties could also depend on the aggregate size [3] and its mineralogic composition [4]. The differences observed in numerical results in comparison with experimental measurements on the mechanical behaviour of concrete are often explained by the fact that the ITZ is not taken into account in the model. Should consideration of the ITZ in modelling be gained?

Standard codes and models used in civil engineering do not take it into account because these models are empirical and the ITZ effects are not of a magnitude relevant to engineering application. Recent numerical models do not allow going down to the fine scale of the ITZ and justify the calculation error by the ITZ effect which cannot be

\footnotetext{
* Corresponding author at: Institut de Recherche en Génie Civil et Mécanique (GeM), UMR-CNRS 6183, Ecole Centrale de Nantes, 1 rue de la Noe, BP 92101, 44321 Nantes cedex 3, France.

E-mail address: frederic.grondin@ec-nantes.fr (F. Grondin).
}

digitalized. Some attempts have been performed to consider the ITZ in numerical models [5-8] and in analytical models [9-12] to calculate the effective properties of concrete as a heterogeneous material. Garboczi and Berryman [7] and Zheng et al. [12] have suggested a homogenization of aggregates with ITZs which then form a new inclusion in the matrix. The homogenization of aggregate + ITZ is unsatisfactory because it leads to a decrease in the strength of the aggregate and it becomes impossible to check the hypothesis of the brittleness of the ITZ which could be the cause of the concrete failure. For modelling the ITZ properties can be calibrated on experimental observations $[4,13,3]$. Many authors have suggested some methods to calculate the ITZ properties by considering some empirical assumptions [14-17], but without linking with the effective behaviour of concrete. Bentz and Garboczi [5] were the only ones who suggest a model which determines the ITZ properties according to the hydration of the cement paste, but the link with the mechanical properties was not established.

In this study, the calculation of the effective behaviour of concrete was performed according to the numerical characterization of the ITZ properties. Two parts of this study are the originality of this work: the calculation of the ITZ mechanical properties according to the hydration of cement and the implementation of the ITZs in a finite element (FE) calculation.

Nadeau [16] has calculated the ITZ properties by supposing an evolution of the water-to-cement $(\mathrm{w} / \mathrm{c})$ ratio according to the distance from the aggregate boundary [18]. By considering this hypothesis, the ITZ properties were calculated according to the age of the concrete, i.e. the cement hydration. The model of Bernard et al. [19], modified by Grondin et al. [20], was retained for the calculation of the cement hydration. The ITZ around an aggregate was supposedly formed by $n$ layers having the same thickness. In each layer the $\mathrm{w} / \mathrm{c}$ ratio is different, so 
the volume fraction of the residual clinkers and formed hydrates was calculated in each layer according to the local hydration kinetic. Then, the effective properties of the ITZs were calculated by homogenization [20] by averaging the local properties of each layers. The homogenization of the ITZ layers keeps the initial strength of the aggregate and allows defining a brittle area between the aggregate and the matrix. This homogenization method does not take into account the brittleness within the layers, but the main goal of this study is the consideration of a brittleness area around aggregate to show its effect on the global behaviour of the specimen.

The ITZ thickness is commonly between $20 \mu \mathrm{m}$ and $50 \mu \mathrm{m}$. In a finite element model it is very numerically expensive to represent it explicitly, even less to decompose it in many layers with different properties. For instance, in a $2 \mathrm{D}$ representative elementary volume (REV) of concrete with dimensions $50 * 50 \mathrm{~mm}^{2}$, if we would represent the ITZ by one finite element with a thickness of $30 \mu \mathrm{m}$, we have to mesh the REV by $2.7 \cdot 10^{6}$ elements. So, in order to take into account the ITZ properties in the calculation with a limitation of the finite elements, we have considered a three-phase material for concrete: aggregates, the bulk paste, and an effective mixed interphase (EMI). The EMI around an aggregate is formed with the ITZ and a part of the bulk cement paste. Its properties are homogenized with the effective properties of ITZs and the bulk cement paste properties according to the volume fractions of ITZs and of the bulk cement paste in EMI. Calculations were performed in the finite element code of calculation Cast $3 \mathrm{~m}$ to assess the damage of concrete with and without ITZs. The damage was obtained by considering a damage model based on the microplane theory [21].

First of all, the algorithm of the model is presented in detail. Second of all, a statistical study is shown in order to define the relevant method to take into account the ITZ properties in the modelling of concrete. Then, the calculation results are analysed for concrete under a tensile load.

\section{Digital representation of the EMI in a FE modelling}

The ITZs surrounding aggregates in concrete could be modelled by an interfacial law in a FE code [22], but we loss the information about the evolution of the properties into the ITZs. Without taking into account the effect of the aggregate size and its mineralogic properties, Nadeau [16] has suggested a model to calculate the volume fraction of cement $f_{\text {citz }}$ through the ITZ at the first contact of cement and aggregates before added water:

$f_{\text {citz }}(r)=f_{c b}\left[1+a_{c}\left(\frac{r-r_{a}-\delta_{I T Z}}{\delta_{I T Z}}\right)^{2}\right]$

where $f_{c b}$ represents the volume fraction of cement in the bulk paste.

The ITZ could be divided in n-layers of thickness equal to $\delta_{I T Z} /\left(r-r_{a}\right)$ and the elastic modulus of the n-layers is calculated according to simple relations linking the elastic moduli of cementitious materials to the $\mathrm{w} / \mathrm{c}$ ratio. Then, the effective elastic modulus of the ITZ can be obtained by averaging the n-layers.

For a detailed modelling of the ITZ influence on the behaviour of concrete, ideally we could represent explicitly each n-layers. But it is easy to notice that a FE modelling is impossible to realize because it needs a too high number of elements due to a high ratio between the specimen volume size and the element size required to represent a layer. Another method could be the modelling of one aggregate in a volume which allows representing all layers. But it is not representative of concrete and it leads to a high approximation of the results.

For a regular FE grid and for a volume size to aggregate size ratio equal to 4 [23], an aggregate maximum size of $12.5 \mathrm{~mm}$ leads to a volume size in $2 \mathrm{D}$ of $50 \times 50 \mathrm{~mm}^{2}$. For instance, even if the ITZ is represented by only one layer of $50 \mu \mathrm{m}$, the finite elements have to be equal to minimum $50 \times 50 \mu \mathrm{m}$ and the total number of elements in the volume is of order $1 \cdot 10^{3} \times 1 \cdot 10^{3}$. So, for the ITZ divided in n-layers the number of finite elements increases substantially. To limit the number of elements, we have considered interphases (EMI) surrounding aggregates containing the ITZ and a volume fraction of the bulk cement paste. The choice of this fraction depends on the interphase thickness:

$\delta_{f b}=\delta_{i n t}-\delta_{I T Z}$

In this study the concrete volume was formed by composite circles (in 2D) with different diameters according to the aggregate size distribution equal to $\varnothing_{\text {agg }}+2 \delta_{\text {int }}$ and a matrix formed by the rest of the bulk cement paste. The generation of the volume was made with the algorithm developed by Mounajed [24] (cited in [23]) and modified to consider the interphase surrounding aggregates (Fig. 1).

The distribution algorithm generates randomly candidate positions of the inclusion gravity centres in the area defined by the REV. At this step, an inclusion $i$ is formed by an aggregate surrounding by an EMI. In $2 D$ the unit volume of an inclusion of type $i$ is calculated as follows:

$V S_{i}=\pi\left(R_{i}^{a g g}+\delta_{i n t}\right)^{2}$.

The unit volume of an aggregate is obtained by:

$V G_{i}=\pi\left(R_{i}^{a g g}\right)^{2}$

Some tests are made to validate these positions. A first test consists to check if this position concerns an element of Digital Concrete type. We calculate the distance between the element gravity centre and that of the inclusion. If this distance is higher than the element average size, this element will have the properties of the inclusion.

A second test checks that two inclusions do not overlap. The distance between an existing inclusion gravity centre, of radius $R 1$, and a candidate inclusion, of radius $R 2$, has to be higher to a value $d_{\min }$ defined as:

$d_{\min }=\left(\frac{R_{1}+R_{2}}{2}\right)$

After the model has validated the inclusions' position, they are affected by characteristic materials defined by the user. Before the inclusions' generation, all the elements were defined by the material parameters of the matrix (the bulk cement paste). Then the model tests if the position of an element gravity centre is in an aggregate, respectively EMI, influence radius. By taking $R^{a g g}$ for the aggregate radius, respectively $\left(R_{i}{ }^{a g g}+\delta_{i n t}\right)$ for the inclusion, and $d_{i n f}$ the distance between an element gravity centre and that of the inclusion, the element takes:

- the aggregate properties if $d_{\text {inf }} \leq R^{\text {agg }}$,

- the EMI properties if $R^{\text {agg }}<d_{\text {inf }} \leq R_{i}^{a g g}+\delta_{\text {int }}$,

- the matrix properties if $d_{i n f}>R_{i}^{a g g}+\delta_{i n t}$.

In order to take into account local properties of the ITZ a homogenization of the n-layers' properties seems necessary. First of all, the relevant number of layers has to be defined to obtain an optimal result. Zheng et al. [12] have suggested a number of layers over 70 but their method was applied to the calculation of the effective properties averaging the modulus of aggregate, the bulk cement paste and the ITZ layers. Here, the homogenization does not consider aggregates. The Voigt's bound was used to calculate the effective modulus of the EMI:

$X^{h o m}=\sum_{k=1}^{n+1} f^{k} X^{k}$

with $f^{k}$ the volume fraction of the layer $k(k=[1, n]$ for the ITZ and $k=n+1$ for the bulk cement paste) with the elastic properties $X^{k}$. The individual properties of each layer were calculated with the model developed by Grondin et al. [20] and modified to take into account the evolution of the properties with the distance from the aggregate surface. 

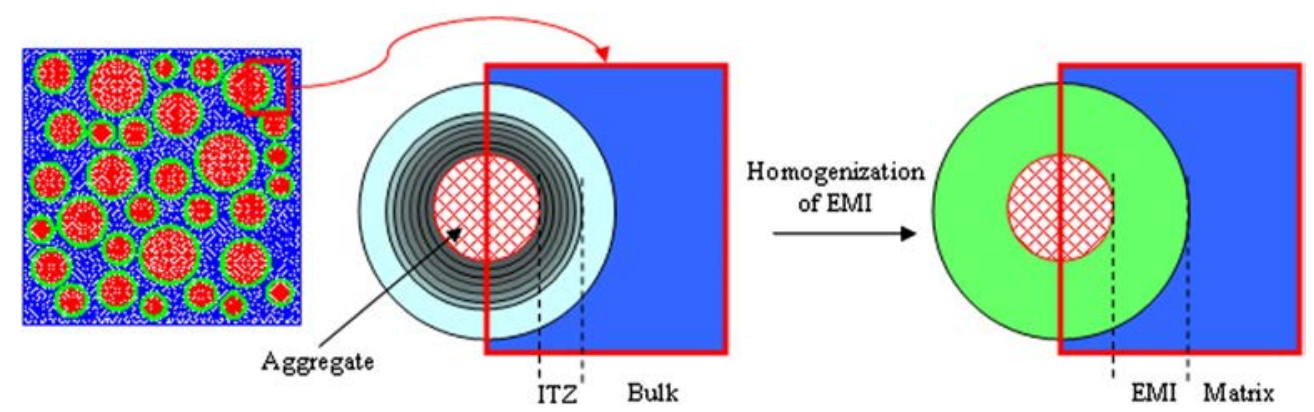

Fig. 1. FE representation of the REV of concrete considering interphases between aggregates and matrix.

The presented model was implemented in the FE code CAST3M [25].

\section{Determination of the ITZ properties according to hydration}

Grondin et al. [20] have suggested a homogenization method, based on the self-consistent scheme, to calculate the elastic properties of cement pastes at early ages according to the hydration progress. The hydration model considers the chemical reactions between clinkers and water according to the initial $\mathrm{w} / \mathrm{c}$ ratio. The calculations are made for each area around aggregates. So for ITZ, we have considered $n$ layers with a specific $\mathrm{w} / \mathrm{c}$ ratio in each layer. The same calculation is made for the bulk cement paste wherein one area is considered. So, in ITZ all hydrates which are formed during the hydration process are considered. The calculations consider implicitly that hydrates fill in the available space.

First of all, the chemical hydration model gives at each time the volume fraction of the residual clinkers and the hydrates. For the layer $k$ the volume of each residual cement phase $\left(V_{x}\right)$ can be calculated as [19]:

$V_{X}^{k}(t)=V_{C 0}^{k} f_{X}^{k}\left(1-\xi_{X}^{k}(t)\right)$

where $V_{C 0}^{k}$ is the initial volume of cement and $f_{X}^{k}$ the initial mass fraction of cement phase $X$. In the same way, the volume of the residual water is equal to:

$V_{E}^{k}(t)=V_{E 0}^{k}-\sum V_{C 0}^{k} \frac{n_{E} \rho_{C} f_{X}^{k} / M_{X}}{n_{X} \rho_{E} / M_{E}} \xi_{X}^{k}(t)$

where $V_{E 0}^{k}$ is the initial volume of water, $M_{X}$ the molar mass of $X, M_{E}$ the molar mass of water and $\frac{n_{E}}{n_{X}}$ the mole number of water $\left(n_{E}\right)$ consumed to hydrate one mole of cement phase $\left(n_{X}\right)$ of the apparent density $\rho_{C} f_{X}^{k}$.

Gypsum is consumed with $C_{3} A$ and $C_{4} A F$ and it is supposed that the consumption kinetic rate of gypsum can be defined by the hydration rate of these two clinkers. So we calculate the volume of gypsum as follows [20]:

$V_{g y p}^{k}(t)=V_{C 0}^{k} f_{g y p}^{k}\left(1-\beta \cdot\left(3 \xi_{C_{3} A}^{k}(t)-3 \xi_{C_{4} A F}^{k}(t)\right)\right)$

where the factor 3 is the stoichiometric coefficient of the chemical reactions and $\beta$ a constant parameter which depends on the $w / c$ ratio. In this study $\beta$ is chosen equal to 2 , respectively 3 , for $w / c$ $\geq 0.4$, respectively $\mathrm{w} / \mathrm{c}<0.4$. This coefficient does not affect the hydration rate but allows to have a uniform consumption of gypsum. Then, when gypsum is totally consumed, ettringite changes in monosulfate and it is supposed the following relation for the volume of ettringite versus time [20]:

$V_{e t t}^{k}(t)=V_{e t t}^{k}\left(t_{g}\right)\left(1-0.5 \xi_{C_{3} A}^{k}(t)-0.5 \xi_{C_{4} A F}^{k}(t)\right)$ where $V_{e t t}^{k}\left(t_{g}\right)$ is the volume of ettringite at the beginning of the formation of monosulfate and the factor 0.5 is the stoichiometric coefficient of the chemical reactions.

The volume of hydrated phases $\left(V_{j}^{P}\right)$ can be calculated according to the volume of elements which have reacted $\left(V_{i}^{R}\right)$ as for the following chemical reaction:

$n_{1}^{R} V_{1}^{R}+\ldots+n_{l}^{R} V_{l}^{R} \rightarrow n_{1}^{P} V_{1}^{P}+\ldots n_{m}^{P} V_{m}^{P}$

we can write:

$V_{i}^{\mathrm{Pk}}(t)=\sum_{j=1}^{l} V_{C 0}^{k} \frac{n_{i}^{P} \rho_{C} f_{j}^{k} / M_{j}}{n_{j}^{R} \rho_{i} / M_{i}} \xi_{j}^{k}(t) \quad i=1, m$.

For the calculation of the elastic properties of the ITZ layers and of the bulk cement paste we have considered a REV $V$ formed by a matrix $V_{m}$ and several types of inclusions, supposed to be spherical and with the same size. Inclusions are defined by their volume fraction in $V$ : $\sum_{i} V_{i}=V_{s}$, where $V_{s}$ is the whole volume of the solid phases in $V$. To calculate the homogenized elastic behaviour, a uniform strain field tensor $\underline{\underline{E}}$ is applied on the volume boundaries. This implies local strain fields $\underline{\underline{\varepsilon}}(\underline{y})$ and local stress fields $\underline{\underline{\sigma}}(\underline{y})$ in each point $\underline{y}$ of $V$ which linked by the following local elastic behaviour law:

$\underline{\underline{\sigma}}(\underline{y})=\underline{\underline{\underline{C}}}^{0}(\underline{y}): \underline{\underline{\varepsilon}}(\underline{y})$

where $\underline{\underline{C}}^{0}$ represents the stiffness tensor of $V$ under drained conditions. By applying the Levin's theorem for the homogenization of elastic heterogeneous media, the resolution of the cellular problem allows to deduce the homogenized behaviour law on the upper scale [26]. The homogenized behaviour law is defined by the relation between the average stresses $\langle\underline{\underline{\sigma}}\rangle_{V}$ and the average strains $\langle\underline{\underline{\varepsilon}}\rangle_{V}$ in each layer $k$ :

$\underline{\underline{\Sigma}}^{k}=<\underline{\underline{\sigma}}^{k}{ }_{V}^{k}=\frac{1}{\left|V^{k}\right|} \int_{V^{k}} \underline{\underline{\sigma}}^{k}(\underline{y}) d y=\underline{\underline{\underline{\underline{C}}}}_{h o m}^{k}:\left\langle\underline{\underline{\varepsilon}}^{k}>_{V^{k}}=\underline{\underline{\underline{\underline{C}}}}_{h o m}^{k}: \underline{\underline{E}}\right.$

where $\underline{\underline{\underline{\underline{C}}}}_{h o m}^{k}$ is the homogenized stiffness tensor which can be defined, for an isotropic elastic behaviour, by the homogenized compressibility and shear moduli, respectively $k^{\text {hom }}$ and $\mu^{\text {hom }}$ :

$\underline{\underline{\underline{\underline{C}}}}^{\text {hom }}=3 k^{\text {hom }} \underline{\underline{\underline{\underline{I}}}}^{\text {vol }}+2 \mu^{\text {hom }} \underline{\underline{\underline{\underline{I}}}}^{\mathrm{dev}}$

$k_{\text {hom }}^{k}$ and $\mu_{\text {hom }}^{k}$ check the following equation system obtained for an isotropic medium:

$\sum_{i=1}^{2} f_{i}^{k} \frac{k_{i}^{k}-k_{\text {hom }}^{k}}{1+\alpha^{k}\left(k_{i}^{k}-k_{\text {hom }}^{k}\right) / k_{\text {hom }}^{k}}=0$ 
$\sum_{i=1}^{2} f_{i}^{k} \frac{\mu_{i}^{k}-\mu_{\text {hom }}^{k}}{1+\beta^{k}\left(\mu_{i}^{k}-\mu_{\text {hom }}^{k}\right) / \mu_{\text {hom }}^{k}}=0$

with:

$\alpha^{k}=\frac{3 k_{\text {hom }}^{k}}{3 k_{\text {hom }}^{k}+4 \mu_{\text {hom }}^{k}}$ and $\beta^{k}=\frac{6\left(k_{\text {hom }}^{k}+2 \mu_{\text {hom }}^{k}\right)}{5\left(3 k_{\text {hom }}^{k}+4 \mu_{\text {hom }}^{k}\right)}$

where $f_{i}^{k}, k_{i}^{k}$ and $\mu_{i}^{k}$ are the volume fractions, the compressibility and the shear moduli of each phases $i$ in each layer $k$.

The model is based on the knowledge of the material component properties. Calculations have been made for a cement of type CEM I 52.5 (Saint Pierre la Cour). The oxide composition has been kept constant: $64.27 \% C_{3} S, 11.06 \% C_{2} S, 13.08 \% C_{3} A, 8.27 \% C_{4} A F$ and $3.32 \% C \bar{S} H_{2}$ with a fineness $\phi=3390 \mathrm{~cm}^{2} / \mathrm{g}$. The kinetic parameters of the cement hydration and the elastic moduli of the cement paste phases have been chosen from the literature review $[19,27]$ and the elastic properties are presented in Table 1. The calculation of the homogenized elastic properties, by applying the self-consistent scheme, needs the knowledge of the elastic properties of all phases. So, we have considered all phases as elastic inclusions and we have characterized air filed pores and water filed pores by a compressibility modulus and a shear modulus. It can be noted for some Young's moduli that their values are between a minimum $\left(E_{\min }\right)$ and a maximum $\left(E_{\max }\right)$. In Table $1, \mathrm{CSH}_{a}$ and $\mathrm{CSH}_{b}$ represent, respectively, the $\mathrm{CSH}$ with a low-density and the $\mathrm{CSH}$ with a high-density. The $\mathrm{CSH}_{a}$ is formed around $\mathrm{C}_{3} S$ and $\mathrm{C}_{2} \mathrm{~S}$ at very early age and the $\mathrm{CSH}_{b}$ is formed around the $\mathrm{CSH}_{a}$ in a second step [37,38].

As written above, the hydration process occurs in three steps: the dissolution, the nucleation and the diffusion. The parameters of the nucleation have been chosen in the literature review [28]. The diffusion depends on the permeability of the $C-S-H$ gel pores surrounding the clinker. Calculations for a material with an initial $\mathrm{w} / \mathrm{c}$ ratio equal to 0.45 have been performed. Figs. 2 and 3 show the evolution of the Young's modulus and the Poisson's coefficient into the ITZ with a thickness of $20 \mu \mathrm{m}$ decomposed in 10 layers of $2 \mu \mathrm{m}$. The Young's modulus, respectively the Poisson's coefficient, increases, and respectively decreases, from layer 1 (the nearest of the aggregate) to layer 10 (the nearest of the bulk cement paste). It is explained by the decrease of the $\mathrm{w} / \mathrm{c}$ ratio from the aggregate to the bulk cement paste.

\section{Influence of the number of layers on the effective behaviour of the EMI}

The Nadeau's model [16] shows that the more that the number of layers is high, the more that the determination of the ITZ properties

Table 1

Elastic moduli of the cement paste phases.

\begin{tabular}{lcl}
\hline Phase & E [GPa] & $v[-]$ \\
\hline $\mathrm{CSH}_{a}$ & $19-23.9$ & 0.25 \\
$\mathrm{CSH}_{b}$ & $27-35$ & 0.25 \\
$\mathrm{CH}$ & $33-48$ & 0.324 \\
$\mathrm{C}_{3} S$ & $128-152$ & 0.314 \\
$\mathrm{C}_{2} \mathrm{~S}$ & $110-150$ & 0.314 \\
$\mathrm{C}_{3} A$ & $110-170$ & 0.314 \\
$\mathrm{C}_{4} A F$ & $100-170$ & 0.314 \\
$\mathrm{C}_{6} A \bar{S}_{3} H_{32}$ & 22.4 & 0.25 \\
$\mathrm{CS}_{2}$ & 45.7 & 0.33 \\
$\mathrm{C}_{4} A \bar{S} H_{12}$ & 42.3 & 0.324 \\
$C_{3}(A, F) H_{6}$ & 22.4 & 0.25 \\
$\mathrm{FH}_{3}$ & 22.4 & 0.25 \\
& & \\
& $\mathrm{k}[\mathrm{GPa}]$ & $\mu[\mathrm{Gpa}]$ \\
\hline Water & 2.2 & 0 \\
Air & 0.141 & 0 \\
\hline
\end{tabular}

is accurate. Figs. 4 and 5 show the influence of the number of layers ( 1 to 10 ) on the values of the effective Young's modulus and the effective Poisson's coefficient of the ITZ with a thickness of $20 \mu \mathrm{m}$ at different ages. The value of the bulk cement paste is given as a reference.

The age of concrete influences only the value of the effective Young's modulus of the ITZ. Its kinetic seems to be the same at each age. The age does not influence the evolution of the effective Poisson's coefficient. So, in this first study on the ITZ we have considered calculations for mature concrete at $72 \mathrm{~h}$. In a future work we will analyze the age influence on the cracking behaviour of concrete.

To determine the optimized number of layers beyond which the effective values of the ITZ do not evolve, we plot the derivation of the Young's modulus according to the number of layers for the ITZ equal to $20 \mu \mathrm{m}$ and $50 \mu \mathrm{m}$ (Fig. 6). For a number of layers beyond 7 , the effective Young's modulus of the ITZ tends to be an asymptote. So, for the following simulations the effective properties of the interphase have been calculated by considering a decomposition of the ITZ in 7 layers.

\section{Application to the study of the tensile behaviour of concrete}

\subsection{The problem formulation}

Tensile tests on concrete specimens have been modelled in order to check the interest to take into account the ITZs at the mesoscopic scale.

A volume $V$ of concrete is formed by two media: a matrix defined by the medium $V_{m}$ and inclusions defined by the medium $V_{i}$. A load $\mathrm{F}$ is applied on one of the surface boundary of $V$ with the unit normal vector $\underline{n}$. This load implies local displacement fields $\underline{u}(\underline{y})$, local strain fields $\underline{\underline{\varepsilon}}(\underline{y})$ and local stress fields $\underline{\underline{\sigma}}(\underline{y})$ in each point $\underline{y}$ of $V$. The non-linear elastic problem is written as follows:

$$
\begin{aligned}
& \underline{\operatorname{div}}(\underline{\underline{\sigma}}(\underline{y})=\underline{0} \quad \forall \underline{y} \in V \\
& \underline{\underline{\sigma}}(\underline{y}=\underline{\underline{\underline{C}}}(\underline{\underline{\varepsilon}}(\underline{y}), \underline{y}): \underline{\underline{\varepsilon}}(\underline{y}) \quad \forall \underline{y} \in V \\
& \underline{\underline{\varepsilon}}(\underline{y})=\frac{1}{2}\left(\underline{\underline{\nabla}} \underline{u}(\underline{y})+{ }^{t} \underline{\underline{\nabla}} \underline{u}(\underline{y})\right) \quad \forall \underline{y} ı n V \\
& \underline{F}=\underline{\underline{\sigma}} \cdot \underline{n} \quad \forall \underline{y} \in \Gamma_{1} \\
& \underline{u}=\underline{U} \quad \forall \underline{y} \in \Gamma_{2}
\end{aligned}
$$

where $\underline{\underline{\underline{C}}}(\underline{\underline{\varepsilon}}(\underline{y}), \underline{y})$ is the secant stiffness tensor depending on the local strains and damage and $\Gamma_{2}$ the boundary of $V$ on which macroscopic displacements conditions are applied.

The chosen damage model is the isotropic model developed by Fichant et al. [21]. It allows to represent the unilateral effect and to obtain objective results independently of the length of the finite elements by controlling the fracture energy. This model is a simplified version of the microplane model of Bazant [29] and is based on the relation between the total stress and the effective stress $\underline{\underline{\sigma}}^{*}(\underline{y})$ of the material defined by:

$$
\underline{\underline{\sigma}}^{*}(\underline{y})=\underline{\underline{\underline{C}}}^{0}(\underline{y}): \underline{\underline{\varepsilon}}^{e}(\underline{y}) \text { or } \underline{\underline{\sigma}}^{*}(\underline{y})=\underline{\underline{\underline{C}}}^{0}(\underline{y}): \underline{\underline{\underline{C}}}^{-1}(\underline{\varepsilon}(\underline{y}), \underline{y}) \underline{\underline{\sigma}}(\underline{y})
$$

where $\underline{\underline{\underline{C}}}^{0}(\underline{y})$ is the initial stiffness of the material phases considered isotropic and linear elastic, and $\underline{\underline{\underline{C}}}^{-1}(\underline{\underline{\varepsilon}}(\underline{y}), \underline{y})$ the compliance tensor 


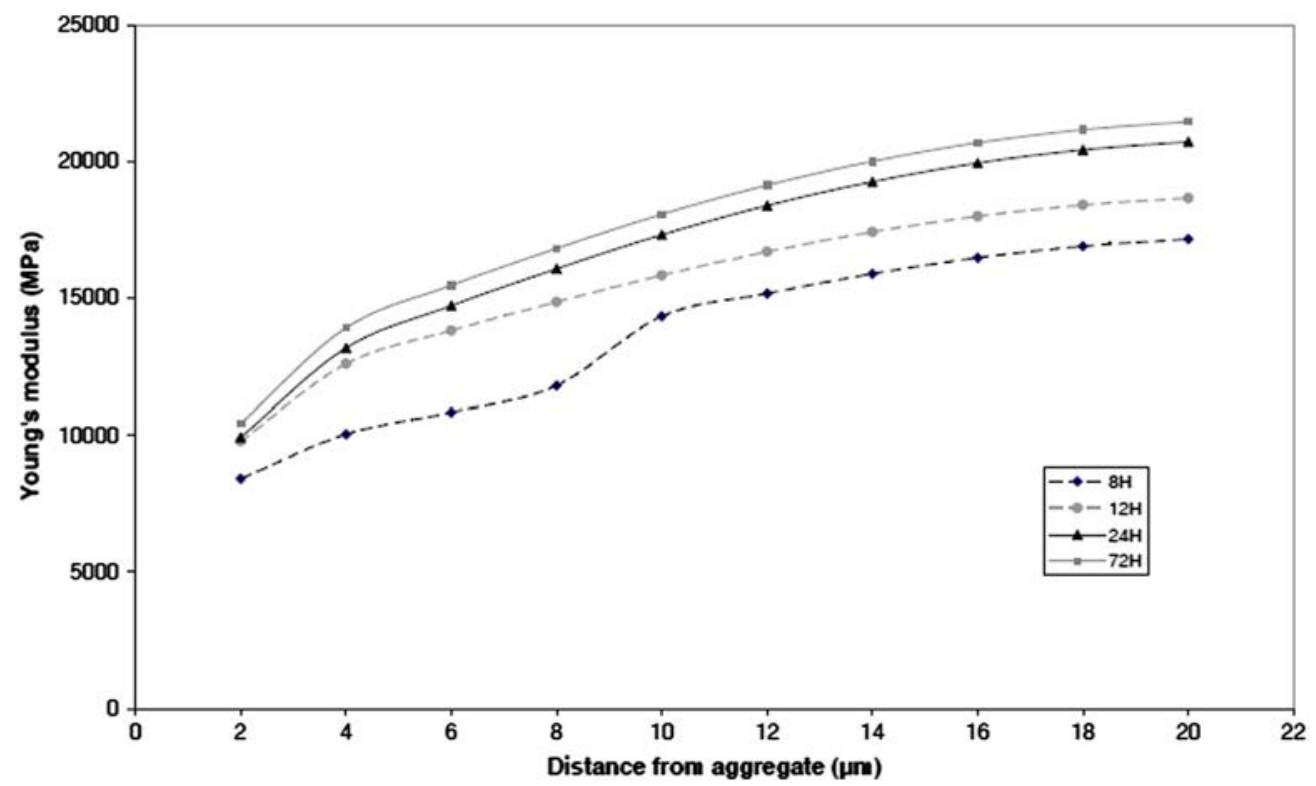

Fig. 2. Variation of the Young's modulus at early ages into ITZ.

of the damaged material. For the isotropic version of the model, the relation between the effective stress and the total stress is given by [30]:

$\underline{\underline{\sigma}}(\underline{y})=(1-d) \underline{\underline{\sigma}} *{ }^{*}(\underline{y})$

where $d$ is the scalar value of the isotropic damage that depends only on the equivalent strain calculated according to the positive part $((\cdot)+)$ of the elastic strain tensor $\underline{\underline{\varepsilon}}^{e}(\underline{y})$ :

$\underline{\underline{\varepsilon}}_{e q}=\sqrt{\left.\left(\underline{\underline{\varepsilon}}^{e}\right)\right)_{+}:\left(\underline{\underline{\varepsilon}}^{e}\right)_{+}}$.
The evolution of the damage variable, due to external mechanical loads, is an exponential form:

$d=1-\frac{\varepsilon_{d 0}}{\varepsilon_{e q}} \exp \left[B_{t}\left(\varepsilon_{d 0}-\varepsilon_{e q}\right)\right]$

where $B_{t}$ represents a damage parameter to control the slope of the strain softening constitutive relation in function of the width $h$ of the element and $\varepsilon_{d 0}$ the strain threshold. Damage increases when the equivalent strain $n_{e q}$ is higher than the threshold strain. The fracture energy $G_{f}$ is given by [31]:

$\frac{G_{f}}{h}=\frac{f_{t} \varepsilon_{d 0}}{2}+\frac{f_{t}}{B_{t}}$

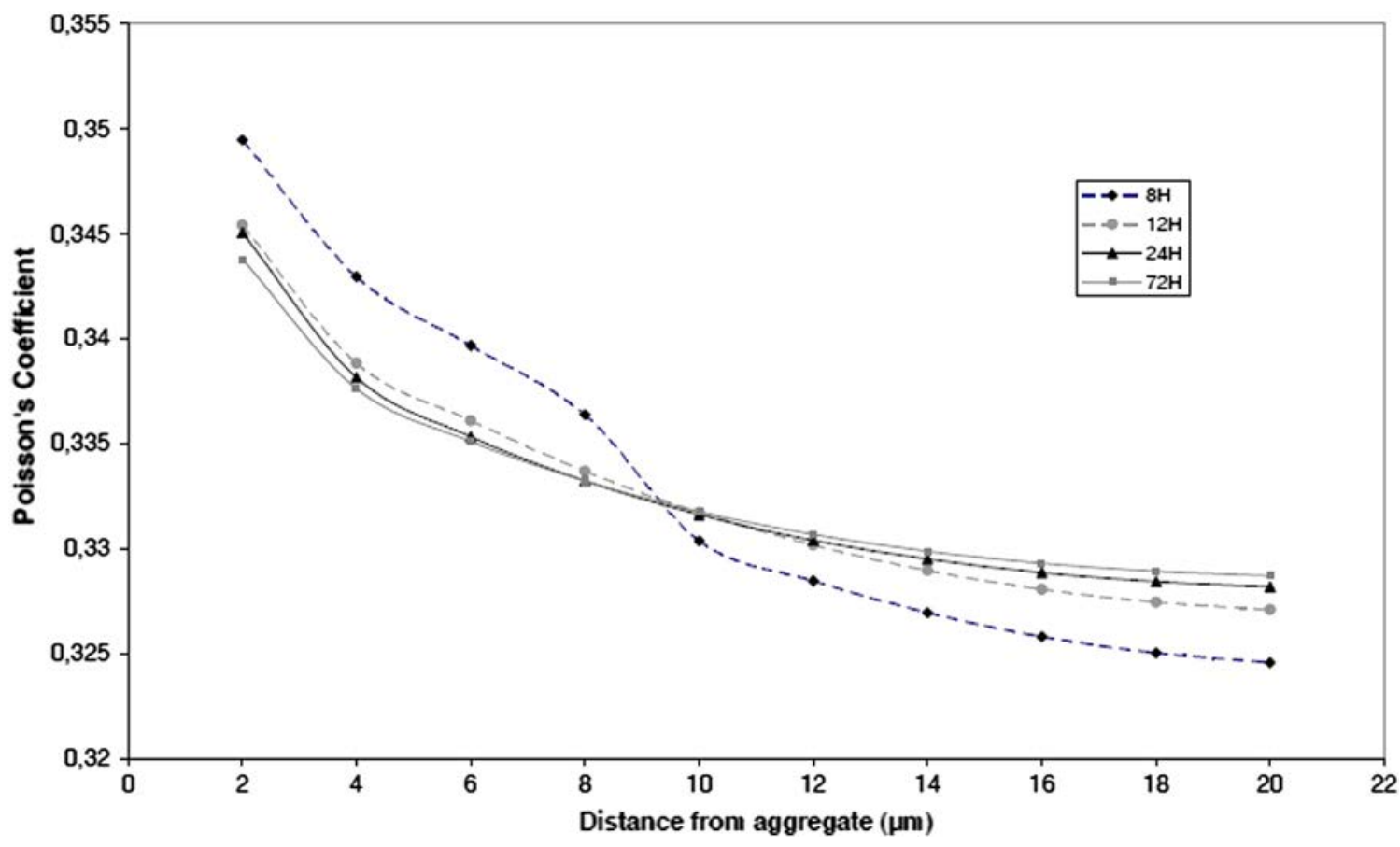

Fig. 3. Variation of the Poisson's coefficient at early ages into ITZ. 


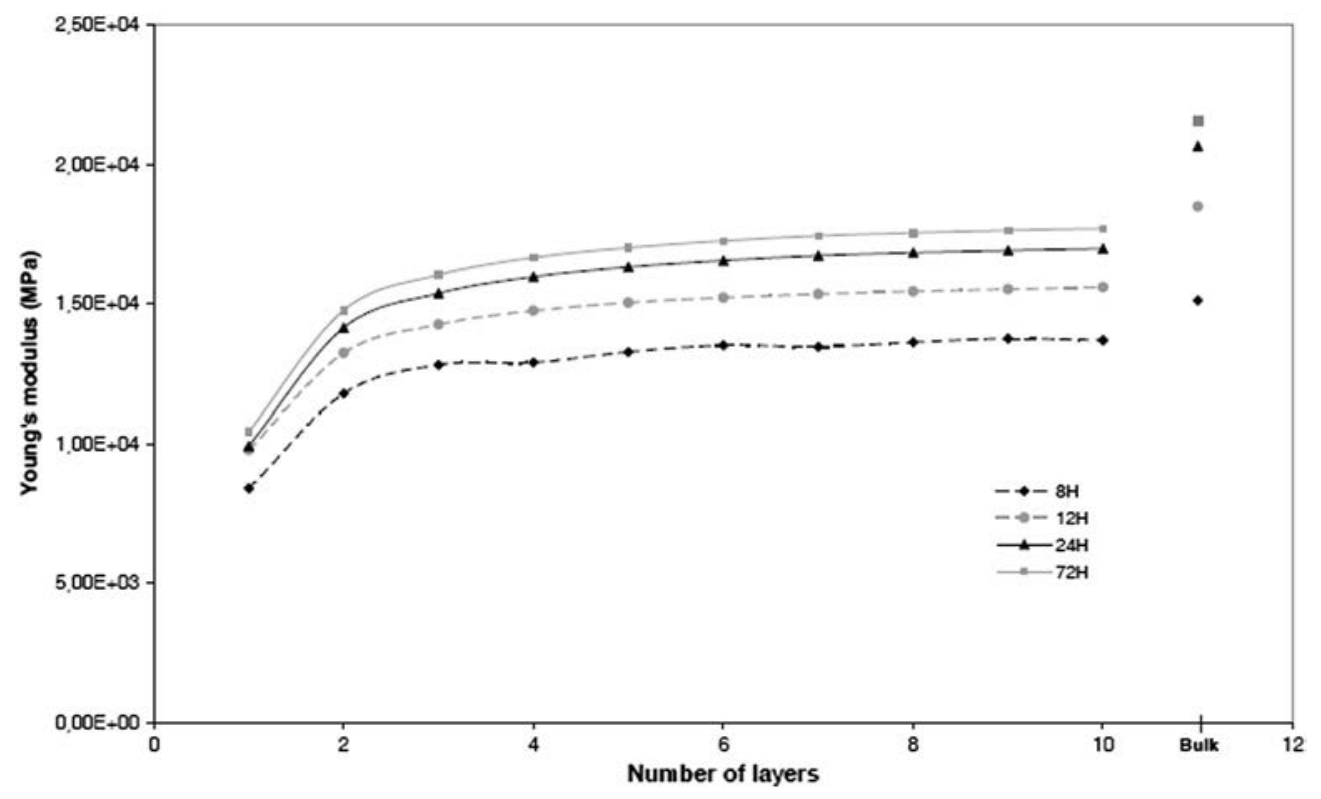

Fig. 4. Influence of the ITZ decomposition on its effective Young's modulus.

where $h$ is the element size and $f_{t}$ the tensile strength. This damage model is based on an energizing regularization issue from the Crack Band Theory where the crack energy monitors the cracking whatever the finite element size [32,31].

\subsection{Analysis of results}

The considered mesoscale for modelling the behaviour of concrete is the scale at which the material can be observed as a set of coarse aggregates embedded in a cementitious matrix. Here, coarse aggregates are inclusions of a size greater than $5 \mathrm{~mm}$ following the experimental aggregate size distribution (Table 3 ) while the matrix is represented by the bulk cement paste. The aggregate volume represents $37 \%$ of the total volume of concrete. The stability of results depending on the size of the specimen and the maximal diameter of the inclusion was performed in a recent study [23] and was respected here with dimensions equal to four times the highest inclusion diameter $(L / D \max >4)$ and finite element size equal to 0.8 time the smallest inclusion diameter. The mechanical properties of the phases are given in Table 2 .

The interphase between aggregates and the cement paste has a thickness of $100 \mu \mathrm{m}$ composed by the ITZ with a thickness equal to $20 \mu \mathrm{m}$, respectively $50 \mu \mathrm{m}$, decomposed in 7 layers for the calculation of its effective properties, and a part of the bulk cement paste with a thickness of $80 \mu \mathrm{m}$, respectively $50 \mu \mathrm{m}$.

Five simulations have been performed for a concrete specimen under a direct tensile load:

- case 1: with the ITZs with a thickness of $20 \mu \mathrm{m}$,

- case 2: with the ITZs with a thickness of $20 \mu \mathrm{m}$ and a lower $f_{t}=2 \mathrm{MPa}$,

- case 3: with the ITZs with a thickness of $50 \mu \mathrm{m}$,

- case 4: with the ITZs with a thickness of $50 \mu \mathrm{m}$ and a lower $f_{t}=2 \mathrm{MPa}$, - case 5: without the ITZs.

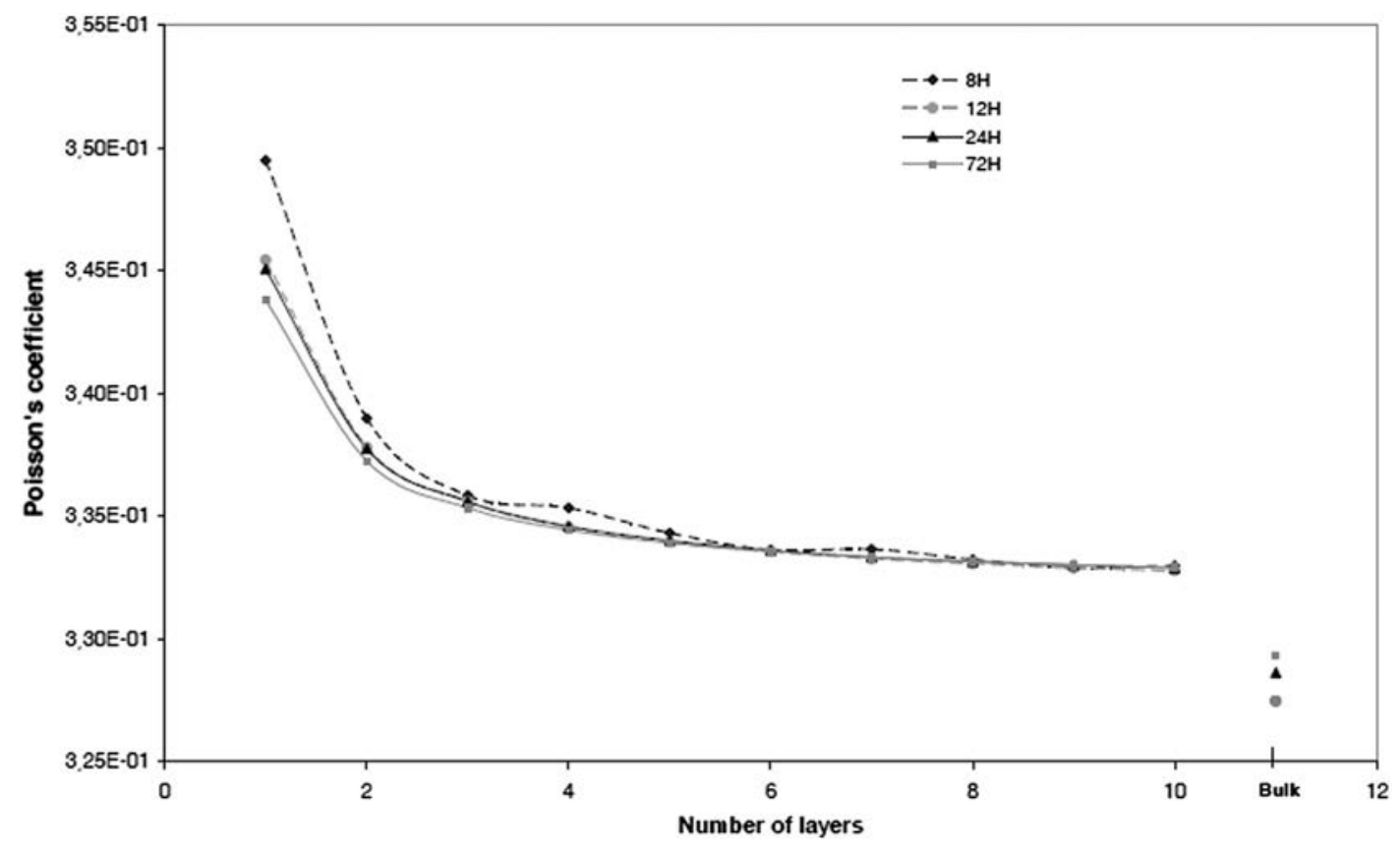

Fig. 5. Influence of the ITZ decomposition on its effective Poisson's coefficient. 


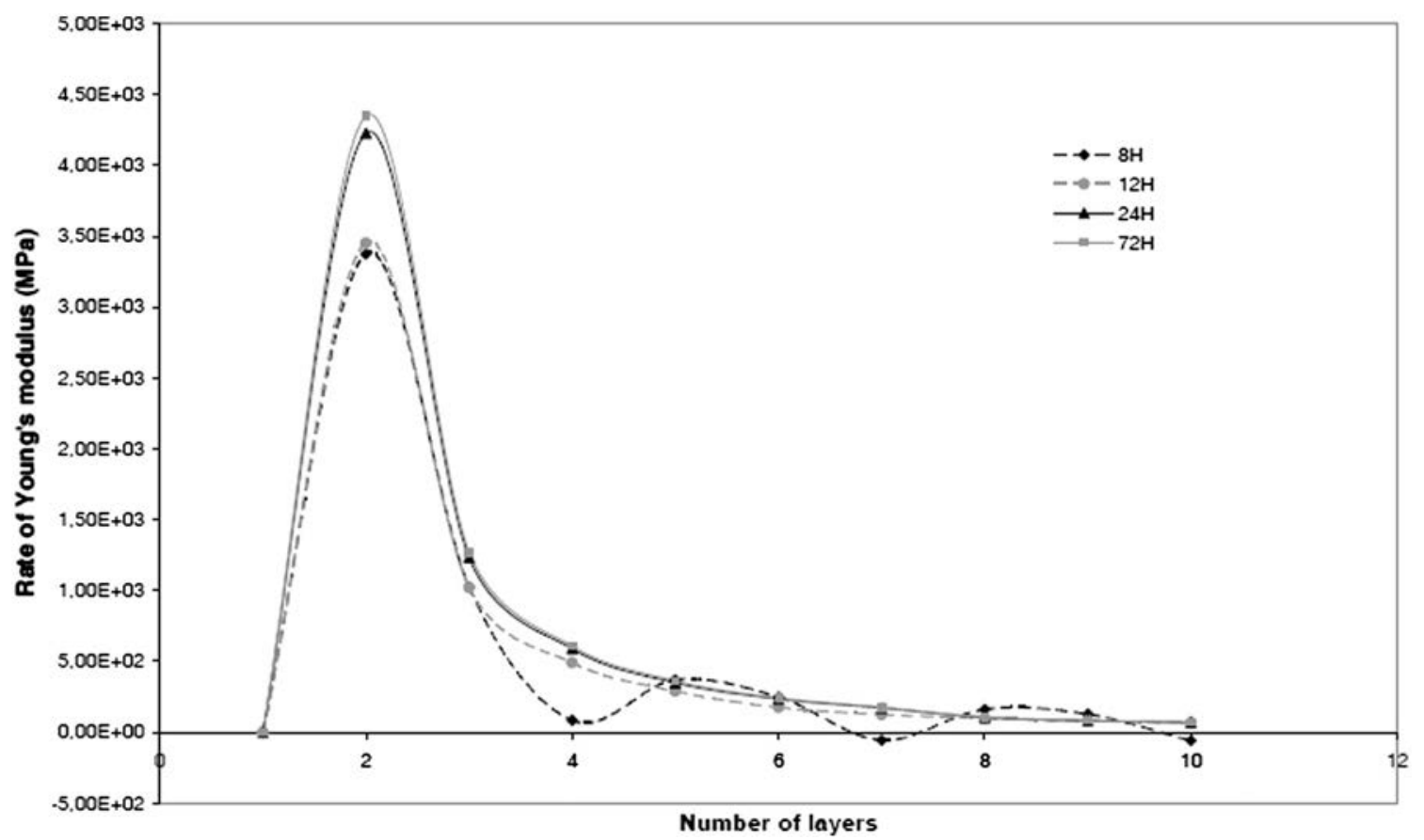

Fig. 6. Rate of the effective Young's modulus vs. the number of layers.

The force-displacement curves are shown in Fig. 7. If the ITZ is not taken into account (case 5), the curve shows a quasi-brittle behaviour with a post-peak region that was not observed in tensile tests [33]. For cases 1 and 3, the elastic part of the curve is the same as that obtained without the ITZs. Also, the thickness is not influent here. But, when damage is considered, the post-peak behaviour varies and cases 1 and 3 show a brittle behaviour as commonly observed in experimental mechanical tests. Cases 2 and 4 have shown a quasi-brittle behaviour with a decrease of the rate of the load before the maximum load was reached which is in good correlation with experimental observations made with the acoustic emission method [34].

The analysis of the local damage fields (Fig. 8) helps to explain the mechanical behaviour. The damage fields are shown at the peak load when the macroscopic crack appears. For case 5, damage is diffused in the microstructure and connectivities between local microcracks are facilitated. So, the macrocracking does not lead to a brittle failure due to a stress diffusion in the matrix. For cases 1 and 3, the diffusion of damage is similar to case 5 but with more localization around aggregates due to a lower elastic modulus. When the macrocracking appears at the maximum load, the stress diffusion is limited. A high density of damage in the macro-crack area leads to quick failure of the specimen. For cases 2 and 4, as the tensile strength of the interphase is lower, damage appears earlier into the interphase and progresses slowly before the maximum load is reached. The macrocracking appears where interphases are close together. Then, the local stresses are redistributed in the bulk cement paste and contribute to a softening behaviour.

Also, one can observe that the macrocrack appears in the same area for cases 2, 4 and 5, between the middle and the bottom of the specimen, and in the same area for cases 1 and 3 , at the top of the specimen

Table 2

The mechanical properties of the concrete phases.

\begin{tabular}{lrlll}
\hline & $E[M P a]$ & $v$ & $G_{f}$ & $f_{t}$ \\
\hline Aggregate & 50 & 0.2 & 80 & 6 \\
Bulk cement paste & 21,590 & 0.33 & 45 & 2.6 \\
EMI $(\mathrm{ITZ}=20 \mu \mathrm{m})$ & 20,324 & 0.33 & 45 & 2.6 \\
EMI $(\mathrm{ITZ}=50 \mu \mathrm{m})$ & 19,595 & 0.33 & 45 & 2.6 \\
\hline
\end{tabular}

close the loading zone. These observations can explain the ability of stress to be diffused into the matrix: limited for cases 1 and 3 , and facilitated for cases 2, 4 and 5 .

Then, the mathematical procedure developed by Matallah et al. [31] to calculate the crack opening according to the damage fields has been applied for the five cases. Results presented in Fig. 9 show the crack opening in concrete. The macro-cracking is well represented where the high density of damage was localized. The crack opening fields show clearly the stress diffusion effect where for cases 1,3 and 5 , the main crack opening has a larger opening than for cases 2 and 4 . The calculation of the crack opening is also interesting for the assessment of the conductivity of concrete. In most of models, calculations overestimate the global permeability of concrete in comparison with experimental measurements. The influence of the microstructure and its evolution are significant [35], so the microstructure has to be better represented in modelling of conductivity by taking into account the ITZs.

\section{Conclusion}

This study has led to few observations and many perspectives:

- The influence of the ITZs is very localized and the choice of their properties has an influence on the effective mechanical behaviour: for modelling at the mesoscopic scale the results are closed to the experimental macroscopic mechanical tests when the ITZs have the same tensile strength of the bulk cement paste, and closed to experimental microstructure inspection tests when the ITZs have a lower tensile strength than the bulk cement paste.

- The various cases studied $(1,2,3,4,5)$ can represent different microstructures of concrete: cases 1 and 3 for concrete with high density ITZ; cases 2 and 4 for concrete with low density ITZ; case 5 for

Table 3

The aggregate size distribution of concrete.

\begin{tabular}{lllrlc}
\hline$\varnothing(\mathrm{mm})$ & 5 & 6.25 & 8 & 10 & 12.5 \\
$V_{f}(\%)$ & 3 & 7 & 11 & 14 & 2 \\
\hline
\end{tabular}




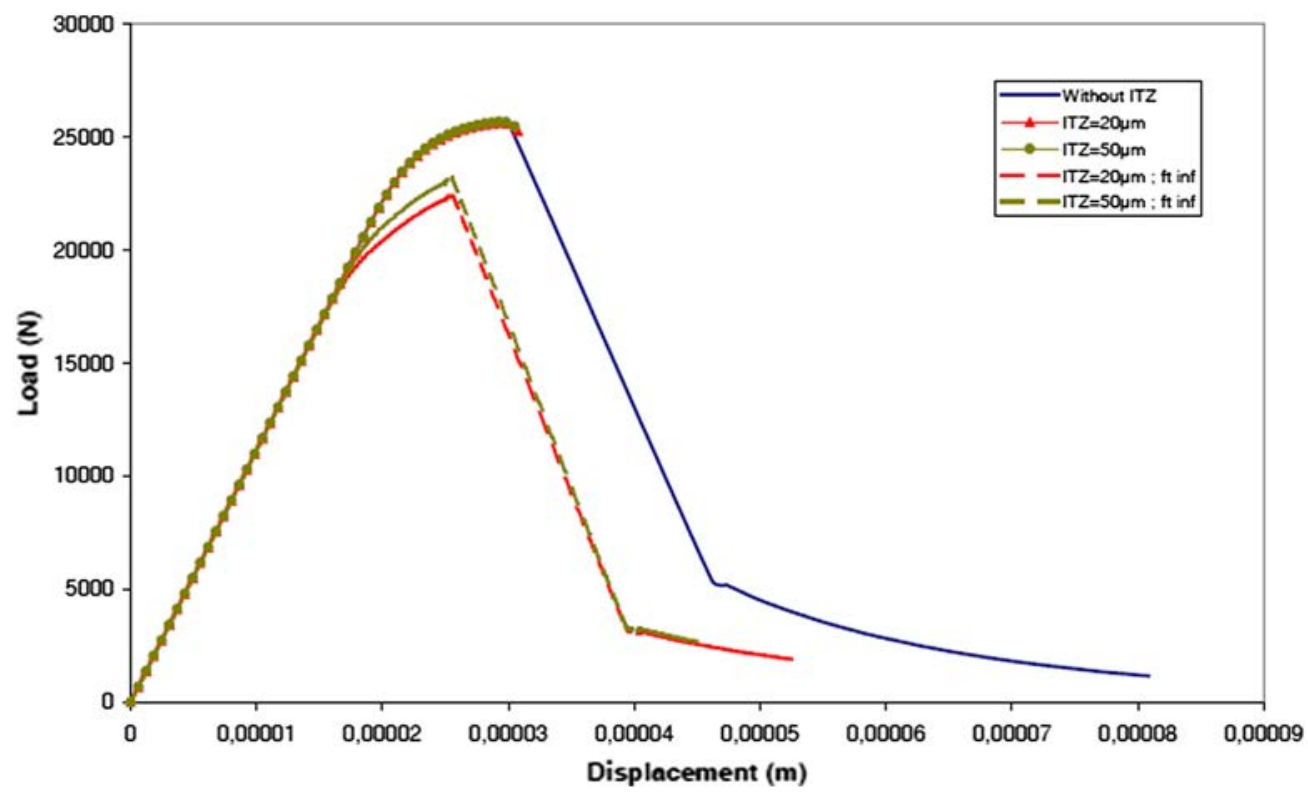

Fig. 7. Force-displacement curves for concrete in tension with different microstructures.

concrete without ITZs. So, without the possibility to take into account the petrology and the morphology of aggregate [4] and their reactivity with the cement paste, the modelling of the microstructure behaviour of various concretes can be performed by taking into account the ITZs with different densities. The local properties into the ITZs have to be compared to experimental measurements as possible and fracture properties could be calibrated on specific experimental tests [36].

- The interest to consider the ITZs in the modelling of concrete has been shown by the results on the crack opening. Because the high density of opened cracks around aggregate can lead to a high increase of the conductivity of concrete and can affect its durability. Also, if a higher volume fraction of aggregate is considered, the connections between opened cracks increases highly.

The main goal of this study was the comparison between two simulations with and without ITZ. The analyses were made according to experimental observations [33] in order to define the relevance of the ITZ presence in a FE mesh. The global displacement-increment used is very small and despite this, brittleness in the softening behaviour is observed. This result may come from the global solving procedure. This paper would show an original method to take into account the ITZs in the modelling of concrete. In future works, sensitivity analyses will be performed in the simulation of cracking, shrinkage and creep of concrete.

The study was focused on the damage of mature concrete at the mesoscopic scale. The age influence has been shown only for the choice of the ITZ properties. A study on the evolution of the age of concrete and its influence on its micro-mechanical behaviour will be performed to follow the cracking progress. Two studies will be performed on the shrinkage at early-ages and on the creep behaviour [33]. Also, in a future work, a diffuse element method around aggregate will be studied by considering a smooth evolution of the interphase properties from the aggregate to the bulk cement paste.
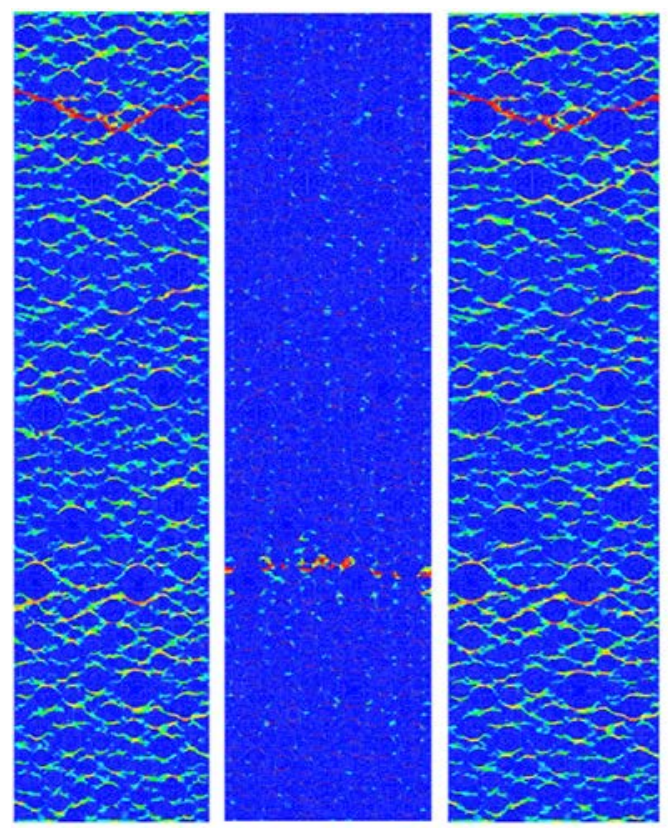

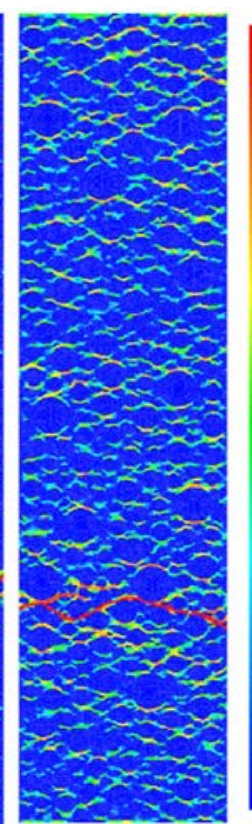

Fig. 8. Damage localization in specimens for the cases (from left to right): 1, 2, 3, 4, 5 . 

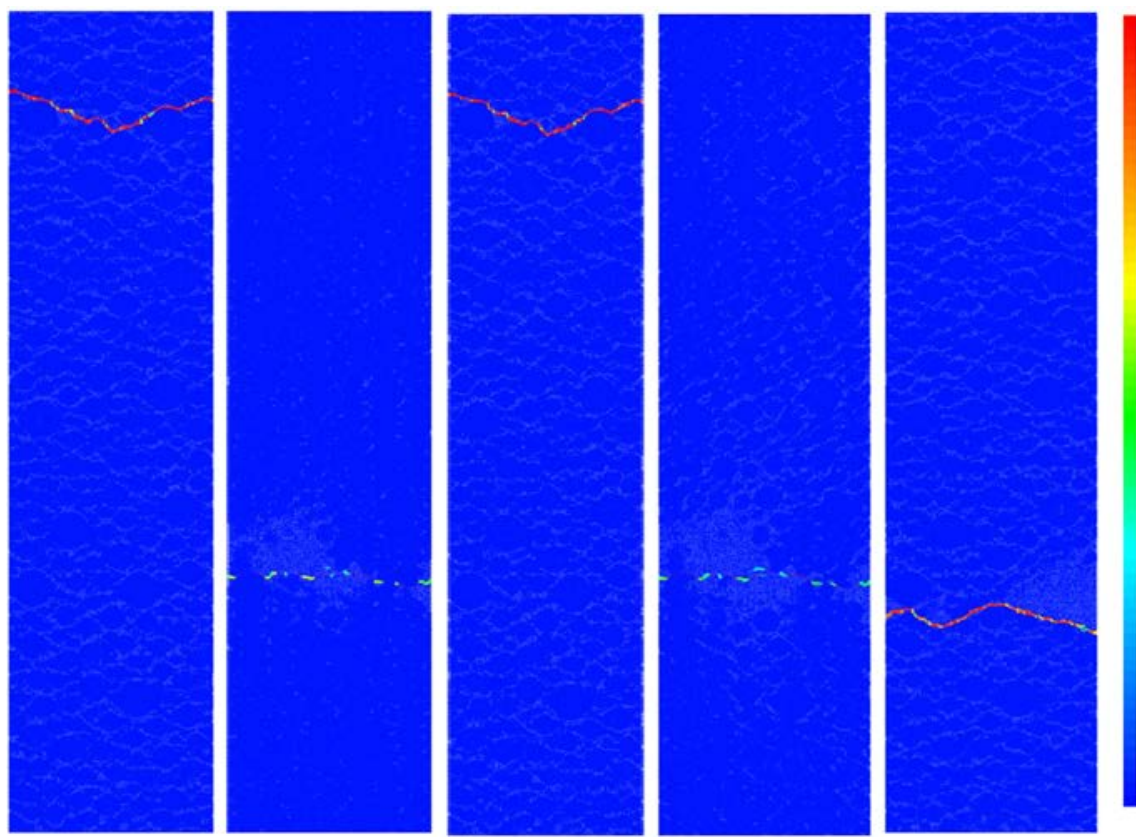

$6.20 \mathrm{E}-05$

5. $90 \mathrm{E}-05$

5. $60 \mathrm{E}-05$

5. $30 \mathrm{E}-05$

$5.00 \mathrm{E}-05$

4. $70 \mathrm{E}-05$

$4.40 \mathrm{E}-05$

4.10E-05

$3.80 \mathrm{E}-05$

$3.50 \mathrm{E}-05$

$3.20 \mathrm{E}-05$

2. $90 \mathrm{E}-05$

2. $60 \mathrm{E}-05$

$2.30 \mathrm{E}-05$

$2.00 \mathrm{E}-05$

1. $70 \mathrm{E}-05$

$1.40 \mathrm{E}-05$

$1.10 \mathrm{E}-05$

$8.00 \mathrm{E}-06$

5. $00 \mathrm{E}-06$

$2.00 \mathrm{E}-06$.

Fig. 9. Crack opening in specimens for the cases (from left to right): 1, 2, 3, 4, 5 .

\section{References}

[1] G. Escadeillas, J. Maso, Approach of the initial state in cement paste, mortar and concrete, Adv. Cement. Mater. (1991) 169-184.

[2] J. Ollivier, J. Maso, B. Bourdette, Interfacial transition zone in concrete, Adv. Cement Based Mater. 2 (1995) 30-38.

[3] A. Elsharief, M. Cohen, J. Olek, Influence of aggregate size, water cement ratio and age on the microstructure of the interfacial transition zone, Cem. Concr. Res. 33 (2003) 1837-1849

[4] W.A. Tasong, C.J. Lynsdale, J.C. Cripps, Aggregate-cement paste interface. Part I. Influence of aggregate geochemistry, Cem. Concr. Res. 29 (1999) 1019-1025.

[5] D. Bentz, E. Garboczi, Computer modelling of interfacial transition zone: microstructure and properties, Engineering and Transport Properties of the Interfacial Transition Zone in Cementitious Composites, Vol. 5, Kluwer Academic Publishers, 1999, pp. 349-385.

[6] E. Garboczi, D. Bentz, J. Shane, T. Mason, H. Jennings, Effect of the interfacial transition zone on the conductivity of portland cement mortars, J. Am. Ceram. Soc. 83 (5) (2000) 1137-1144.

[7] E. Garboczi, J. Berryman, New effective medium theory for the linear elastic moduli of a material containing composite inclusions, Mech. Mater. 33 (2001) 455-470.

[8] K.M. Lee, J.H. Park, A numerical model for elastic modulus of concrete considering interfacial transition zone, Cem. Concr. Res. 38 (2008) 396-402.

[9] C. Neubauer, H. Jennings, E. Garboczi, A three-phase model of the elastic and shrinkage properties of mortar, Adv. Cement-Based Mater. 4 (1996) 6-20

[10] E. Garboczi, Stress, displacement, and expansive cracking around a single spherica aggregate under different expansive conditions, Cem. Concr. Res. 27 (4) (1997) 495-500.

[11] J. Nadeau, A multiscale model for effective moduli of concrete incorporating ITZ water-cement ratio gradients, aggregate size distributions, and entrapped voids, Cem. Concr. Res. 33 (2003) 103-113.

[12] J. Zheng, X. Zhou, X. Jin, An n-layered spherical inclusion model for predicting the elastic moduli of concrete with inhomogeneous ITZ, Cem. Concr. Compos. 34 (2012) 716-723.

[13] S. Diamond, J. Huang, The ITZ in concrete-a different view based on image analysis and sem observations, Cem. Concr. Compos. 23 (2001) 179-188.

[14] B. Bourdette, E. RIngot, J.P. Olivier, Modelling of the transition zone porosity, Cement Concr. Res. 25 (4) (1995) 741-751.

[15] Z. Hashin, P. Monteiro, An inverse method to determine the elastic properties of the interphase between the aggregate and the cement paste, Cem. Concr. Res. 32 (2002) 1291-1300.

[16] J. Nadeau, Water-cement ratio gradients in mortar and corresponding effective elastic properties, Cem. Concr. Res. 32 (2002) 481-490.

[17] Y. Ke, A.L. Ortola, S. Beaucour, H. Dumontet, Identification of microstructural charac teristics in lightweight aggregate concretes by micromechanical modelling including the interfacial transition zone (ITZ), Cem. Concr. Res. 40 (2010) 1590-1600.

[18] K. Scrivener, P. Pratt, Characterization of interfacial microstructure, RILEM Report, 11, E \& FN Spon, 1996, pp. 1-17.

[19] O. Bernard, F. Ulm, E. Lemarchand, A multiscale micromechanics-hydration mode for the early-age elastic properties of cement-based materials, Cem. Concr. Res. 33 (2003) 1293-1309.
[20] F. Grondin, M. Bouasker, P. Mounanga, A. Khelidj, Physico-chemical deformations of solidifying cementitious systems: multiscale modelling, Mater. Struct. 43 (1) (2010) 151-165.

[21] S. Fichant, G. Pijaudier-Cabot, C. Laborderie, Continuum damage modeling: approximation of crack induced anisotropy, Mech. Res. Commun. 24 (2) (1997) 109-114.

[22] E. Gal, E. Suday, H. Waisman, Homogenization of materials having inclusions surrounded by layers modeled by the extended finite element methods, Int. J. Multiscale Comput. Eng. 11 (3) (2013) 239-252.

[23] F. Grondin, H. Dumontet, A. Ben Hamida, G. Mounajed, H. Boussa, Multi-scales modelling for the behaviour of damaged concrete, Cem. Concr. Res. 37 (2007) $1453-1462$.

[24] G. Mounajed, Exploitation du nouveau modèle béton numérique dans symphonie: Concept, homogénéisation du comportement thermomécanique des bhp et simulation de l'endommagement thermique, Cahiers du CSTB, 3421, CSTB, 2002.

[25] P. Verpaux, T. Charras, A. Millard, Une approche moderne du calcul des structures, Pluralis, 1988.

[26] L. Dormieux, A. Molinari, D. Kondo, Micromechanical approach to the behavior of poroelastic materials, J. Mech. Phys. Solids 50 (2002) 2203-2231.

[27] C.-J. Haecker, E. Garboczi, J. Bullard, R. Bohn, Z. Sun, S. Shah, T. Voigt, Modeling the linear elastic properties of portland cement paste, Cem. Concr. Res. 35 (10) (2005) $1948-1960$

[28] R. Berliner, M. Popovici, K. Herwig, M. Berliner, H. Jennings, J. Thomas, Quasielastic neutron scattering study of the effect of water-to-cement ratio on the hydration kinetics of tricalcium silicate, Cem. Concr. Res. 28 (2) (1998) 231-243.

[29] Z. Bazant, Microplane model for strain controlled inelastic behaviour, Mech. Eng. Mater. (1984) 45-59.

[30] S. Fichant, C. Laborderie, G. Pijaudier-Cabot, Isotropic and anisotropic descriptions of damage in concrete structures, Mech. Cohesive-Frict. Mater. 4 (4) (1999) 339-359.

[31] M. Matallah, C. Laborderie, O. Maurel, A practical method to estimate crack openings in concrete structures, Int. J. Numer. Anal. Methods Geomech. 34 (2010) 1615-1633.

[32] M. Jirasek, M. Bauer, Numerical aspects of the crack band approach, Comput. Struct. 110-111 (2012) 60-78.

[33] J. Saliba, F. Grondin, M. Matallah, A. Loukili, H. Boussa, Relevance of a mesoscopic modelling for the coupling between creep and damage in concrete, Mechanics of time-dependent materials 17 (3) (2013) 481-499.

[34] Y. Zhang, O. Abraham, F. Grondin, A. Loukili, V. Tournat, A. Le Duff, B. Lascoup, O. Durand, Study of stress-induced velocity variation in concrete under direct tensile force and monitoring of the damage level by using thermally-compensated coda wave interferometry, Ultrasonics 52 (8) (2012) 1038-1045.

[35] F. Grondin, H. Dumontet, A. Ben Hamida, H. Boussa, Micromechanical contributions to the behaviour of cement-based materials: two-scale modelling of cement paste and concrete in tension at high temperatures, Cem. Concr. Compos. 33 (3) (2011) 424-435.

[36] M. Matallah, M. Farah, F. Grondin, A. Loukili, E. Roziére, Size-independent fracture energy of concrete at very early ages by inverse analysis, Eng. Fract. Mech. 109 (2013) 1-16.

[37] H.M. Jennings, A model for the microstructure of calcium silicate hydrate in cement paste, Cem. Concr. Res. 30 (1) (2000) 101-116.

[38] P.D. Tennis, H.M. Jennings, A model for two types of calcium silicate hydrate in the microstructure of portland cement paste, Cem. Concr. Res. 30 (6) (2000) 855-863. 